\title{
Experiência de mentoria na residência médica: desafios e vivências
}

\author{
Mentoring experience in medical residency: challenges and experiences
}

\author{
Fernando Lima Lopes Filho' ${ }^{10} \mid$ flimalopesf@gmail.com \\ Francisco Vileimar Andrade de Azevedo' (D) vileimarandrade@gmail.com \\ Kristopherson Lustosa Augusto 2,3,4 (D) kristopherson@ufc.br \\ Hyvinna Suellen de Oliveira Silveira² (D) hyvinnasilveira@gmail.com \\ Fabrício André Martins da Costa ${ }^{1,2}$ (1) fabricioamc18@gmail.com \\ Karine Paschoal Botelho Alves ${ }^{1}$ (D) dermato@karinebotelho.com
}

\begin{abstract}
RESUMO
Introdução: A residência médica representa um momento desafiador, com elevado nível de exigência e necessidade de desenvolvimento de inúmeras competências pessoais e profissionais. Com base nisso, implantou-se, em abril de 2019, um programa de mentoria inédito para 30 residentes de clínica médica, buscando oferecer suporte pessoal e estimular desenvolvimento profissional.

Relato de experiência: O programa de mentoria foi estruturado em abril de 2019, com 30 residentes de clínica médica e seis mentores com experiência em preceptoria clínica. Com duração de 11 meses, o programa abordou temas não usualmente discutidos na formação desses especialistas, como profissionalismo, empatia, burnout e qualidade de vida, gestão de tempo, gestão de conflitos e role-model. Os encontros ocorriam mensalmente, com local definido pelo grupo. Após o fim da atividade, foi realizada pesquisa de opinião utilizando questionário padronizado com respostas em escala Likert para avaliação de relevância dos temas discutidos e impacto na prática dos médicos residentes. Obtiveram-se $90 \%$ de respostas, nas quais se destacou a relevância dos temas burnout e qualidade de vida e empatia. Dos residentes, 96,3\% afirmaram que as discussões mudaram a forma deles de pensar e agir profissionalmente. Destacaram que o mentoring "abria a mente acerca de diversidade de opiniões, permitindo crescimento pessoal e profissional" e que "Gerir o tempo, conflitos, burnout estão presentes no nosso dia a dia e também fazem parte da medicina que praticamos", pontuando dificuldade de consenso entre os membros do grupo para definição do horário e dia a ser realizada a reunião. A atividade demonstrou avaliação positiva dos mentorados.
\end{abstract}

Discussão: Na formação de um médico residente, o programa de mentoria melhora a autoconfiança e promove um impacto positivo na carreira profissional apesar das dificuldades enfrentadas. A mentoria funciona ainda como suporte psicossocial, pois cria-se um livre acesso entre residente e preceptor, o que culmina, na nossa avaliação, em uma excelente relação entre mentores e mentorados.

Conclusão: No Ceará, o programa de mentoring para os residentes de clínica médica foi inédito. Houve efeito positivo, em que temas importantes foram discutidos com mais profundidade, com maior crescimento pessoal e profissional. Os autores sugerem que o mentoring seja mantido e incluído como atividade essencial no currículo dos programas de residência médica.

Palavras-chave: Educação Médica; Mentoria; Residência Médica.

\section{ABSTRACT}

Introduction: Medical residency represents a challenging moment, with a high level of demand and the need to develop several personal and professional skills. Therefore, in April 2019, the unprecedented mentoring program for thirty internal medicine residents was implemented, seeking to offer personal support and encourage professional development.

Experience Report: A mentoring program was structured in April 2019, lasting 11 months, consisting of 30 internal medicine residents and 6 mentors with experience in clinical preceptorship, covering topics that are not usually discussed in the training of these specialists, such as professionalism, empathy, burnout and quality of life, time management, conflict management and role-model. The meetings took place monthly, in a location defined by the group. After the end of the activity, an opinion poll was conducted using a standardized questionnaire with answers arranged on a Likert scale to assess the relevance of the discussed topics and the impact on the medical residents' practice. A 90\% response rate was obtained, highlighting the relevance of the topic Burnout and quality of life and empathy. A total of 96.3\% of residents stated that the program changed their mind about their professional behavior. They declared that mentoring "opened their mind about the diversity of opinions, allowing personal and professional growth" and that "to manage time, conflicts, burnout are present in our daily lives and are also part of the medicine we practice". However, there were complaints about some difficulties among the group members to define the time and day when the meetings should be held. In summary, the activity had a positive evaluation from the mentees.

Discussion: There is a positive impact of the mentoring program on the training of a resident doctor, improving self-confidence and having a positive impact on the professional career despite the faced difficulties. Mentoring also serves as a psychosocial support, creating free access between the resident and the preceptor, culminating, based on our evaluation, in an excellent relationship between mentors and mentees.

Conclusion: In Ceará, the mentoring program for internal medicine residents was unprecedented. There is a positive effect, in which important topics were discussed in more depth, with greater personal and professional growth. We suggest that mentoring be maintained and included as an essential activity in the curriculum of Medical Residency Programs.

Keywords: Medical Education; Mentoring; Medical Residency.

${ }^{1}$ Hospital Geral Doutor Waldemar Alcântara, Fortaleza, Ceará, Brasil.

${ }^{2}$ Universidade de Fortaleza, Fortaleza, Ceará, Brasil.

${ }^{3}$ Centro Universitário Christus, Fortaleza, Ceará, Brasil.

${ }^{4}$ Universidade Federal do Ceará, Fortaleza, Ceará, Brasil.

Editora-chefe: Rosiane Viana Zuza Diniz

Editora associada: Izabel Cristina Meister Martins Coelho

Recebido em 04/03/21; Aceito em 23/04/21. | Avaliado pelo processo de double blind review. 


\section{INTRODUÇÃO}

$A$ residência médica representa um período com elevado nível de exigência profissional, sendo um momento único e bastante desafiador. Ao vivenciar um ambiente com elevado nível de exigência, o médico residente deve desenvolver competências dentro da sua especialidade ao mesmo tempo que lida com inúmeros fatores de estresse, como um deficiente sistema assistencial público, extensa carga horária e privação de sono, que trazem prejuízos para a sua saúde mental, tornando-o menos capaz de prover cuidado adequado e seguro aos seus pacientes ${ }^{1,2,4}$.

Diante disso, programas de tutoria, na forma de mentoring, têm sido propostos como uma estratégia para oferecer suporte pessoal e estimular o desenvolvimento profissional dos futuros especialistas ${ }^{1,2}$. Nessa modalidade de ensino, há uma relação empática entre o médico residente, o mentorado, e seu mentor, um médico experiente que auxilia nos diferentes desafios ao longo da formação, representando uma figura que inspira e guia ${ }^{4,5}$.

Os programas de mentoria proporcionam ao mentorado crescimento pessoal e desenvolvimento profissional, com a função de abordar temas relacionados à carreira médica, ao bem-estar psicossocial e ao role-model. Em um estudo que examinou 24 escolas médicas, os indivíduos mentorados apresentaram significativamente mais satisfação profissional do que aqueles que não possuíam mentor ${ }^{3}$. Além disso, o mentoring foi fortemente associado a um melhor desempenho em exames de certificação de especialidade nos Estados Unidos².

Dessa forma, a implantação de um plano de formação médica capaz de unir o desenvolvimento de habilidades técnicas e conhecimento específico ao desenvolvimento afetivo, social e ético, com contato próximo entre mentor e mentorado, causará impacto positivo na saúde mental do médico residente, contribuindo para a melhora da autoconfiança desse profissional, de modo a torná-lo mais seguro para exercer, inclusive, o papel de mentor no processo de ensino-aprendizagem para acadêmicos e internos de medicina. É dessa forma que se estrutura uma rede de apoio institucional ${ }^{1-4}$.

Este relato traz a experiência inédita de um programa de mentoria desenvolvido para a residência de clínica médica em um hospital secundário, localizado no estado do Ceará, em que descrevemos as etapas de sua estruturação e implementação, e avaliamos, por meio da aplicação de questionário padronizado, os impactos práticos dos temas e das estratégias discutidos na vida cotidiana do médico residente.

\section{RELATO DE EXPERIÊNCIA}

\section{Estruturação, implementação e organização do programa de mentoria}

Em 2018, um grupo de preceptores participou de uma oficina de mentoria em um congresso médico, surgindo então a ideia de instituir o mentoring na residência médica. Em abril de 2019, após reunião entre a Comissão de Residência Médica (Coreme) e a Coordenação da Residência de Clínica Médica, era instituído o programa de mentoria voltado para 30 residentes de clínica médica, com duração de 11 meses. Escolheu-se um grupo de mentores de acordo com o interesse dos preceptores do serviço. Após essa escolha, o grupo foi capacitado sobre a técnica do mentoring, o que ocorreu com a colaboração de uma equipe de médicos mentores com experiência em um hospital universitário de outro estado. A capacitação ocorreu por meio de encontros realizados por videoconferência, nos quais uma equipe de tutores relatou as próprias experiências de mentoria na residência médica e transmitiu alguns conceitos teóricos e noções práticas de organização do método.

O grupo de mentores definiu, então, que o mentoring deveria ser estruturado em grupos compostos de um mentor e cinco mentorados. Nesse sentido, o mentor deveria ser, invariavelmente, médico com experiência em preceptoria de clínica médica, além de ter disponibilidade para realização de encontros mensais. Era imprescindível que o mentor possuísse características como empatia, espírito de liderança e desejo de compartilhar suas experiências com o grupo de mentorados, contribuindo para uma evolução pessoal e profissional deles. Quanto aos mentorados, seriam médicos residentes de clínica médica componentes do primeiro e segundo anos de especialização.

Os temas a serem discutidos foram definidos previamente pelos mentores e coordenadores da residência, sendo eles: profissionalismo, burnout e qualidade de vida, empatia, role model, gestão de conflitos e gestão de tempo.

Cada tema deveria ser introduzido pelo mentor, no entanto dois mentorados estariam encarregados de possuir maior embasamento teórico do tema em foco, com referências científicas. O mentor, inicialmente, colocava em pauta alguns questionamentos para iniciar a reunião, que posteriormente transcorria livremente, da forma como os mentorados desejassem ou se manifestassem.

Os encontros ocorreram mensalmente, com a atividade sendo categorizada como prioritária e obrigatória dentro da carga horária da residência médica. O local e o horário eram definidos em conjunto pelo grupo a partir dos interesses dos seus componentes, com diversos encontros ocorrendo em locais fora do ambiente hospitalar, para que houvesse uma atmosfera de maior relaxamento e descontração, como restaurantes ou cafés. $O$ tempo de duração da atividade era de duas horas. Durante a discussão, questionamentos e reflexões eram gerados e debatidos em conjunto, sendo formado um ambiente no qual os residentes poderiam falar livremente, com segurança e sem cobranças da rotina profissional. 


\section{Feedback dos mentorados}

Ao fim da atividade, em fevereiro de 2020, realizouse uma pesquisa de opinião a respeito dos temas discutidos com todos os mentorados, na qual se utilizou um questionário padronizado com respostas em escala Likert de 6 graduações (0 a 5), com respostas categorizadas que vão de "concordo totalmente" a "discordo totalmente", por meio da plataforma Google Forms, de forma anônima. Desenvolvemos questões para avaliação do quão importantes foram os temas discutidos e graduamos as respostas numa escala de 0 a 5 pontos, sendo 0 considerado "sem importância" e 4 e 5 com "muita importância".

Para entendimento da relevância do programa de mentoria para cada residente, o questionário conteve as seguintes perguntas: "Os temas discutidos foram relevantes para a sua prática profissional?", "O programa de mentoring mudou sua conduta profissional médico/residente?" e "Quais dos temas escolhidos tiveram maior potencial de mudança na sua atuação como profissional de saúde?". Foi solicitado ainda que cada residente sugerisse um tema para compor as discussões, bem como as melhorias que poderiam ser implementadas para aprimoramento da estrutura e dinâmica da atividade. Obtivemos $90 \%$ de respostas ao questionário, um total de 27 residentes $(n=30)$.

Uma análise quantitativa mostrou que $44,4 \%$ dos residentes concordaram totalmente que as discussões desenvolvidas mudaram a forma deles de pensar e agir como profissionais, enquanto $51,9 \%$ concordaram de maneira parcial. Quando analisamos de forma qualitativa esse resultado, solicitando que justificassem seu posicionamento, obtivemos inúmeras avaliações positivas:

Ajudam a abrir a cabeça para assuntos que não costumamos separar um tempo para discutir e que são essenciais a nossa rotina de médico e de pessoa.

Discutir sobre temas do mentoring abriram nossa mente acerca de opiniões diversas e nos permitiu um crescimento pessoal e profissional, além de ter ajudado a acolher e nos sentir acolhidos nas nossas dificuldades.

As discussões abordavam temas que fazem parte da prática médica, mas que não nos ensinam na faculdade. Gerir o tempo, conflitos, burnout estão presentes no nosso dia a dia e também fazem parte da medicina que praticamos. Falar sobre e ouvir os conflitos dos outros nos ensina a abordar melhor esses problemas no dia a dia.

A mentoria permitia que os residentes fossem colocados, diante de seu staff, em uma posição de horizontalidade, podendo, naquele momento, se posicionar de forma mais livre e natural, possibilitando que temas importantes, mas usualmente não desenvolvidos, fossem tratados com profundidade e naturalidade. Isso fez com que grande parte do que era discutido ficasse fixado.

Alguns residentes citaram pontos negativos: "Com o passar do tempo e as dificuldades do dia a dia, as ideias discutidas não são muito factíveis".

Além disso, houve o seguinte comentário: "Os grupos são heterogêneos pela diferença do mentor. Talvez tentar padronizar um mínimo de atividades para não ficar tão discrepante a experiência entre os residentes".

Ademais, quando perguntados sobre mudança específica de conduta como médico residente em algum aspecto, $81,4 \%$ concordaram que houve impacto (37\% totalmente e $44,4 \%$ parcialmente). Quando questionados a respeito da relevância dos temas propostos para discussão, $96,3 \%$ concordaram que eles são relevantes para a prática médica.

Em relação às temáticas mais relevantes para as reuniões, burnout foi o tema de maior impacto para os mentorados em termos de modificação do campo de prática, recebendo notas 4 e 5 para $77,8 \%$ dos médicos residentes, dos quais $51,9 \%$ deram o valor máximo e $25,9 \%$ atribuíram nota 4 .

Logo em seguida, o tema empatia foi o segundo com maior relevância, apresentando um percentual de 70,3\% (40,7\% deram nota 4 e $29,6 \%$ atribuíram nota máxima). 0 tema gestão de conflitos foi relevante e modificador de prática para $66,6 \%$ dos residentes, seguido por profissionalismo, com $59,3 \%$ de avaliação positiva. Os temas gestão de tempo e role-model apresentaram, respectivamente, $51,9 \%$ e 48,1\% (Tabela 1).

Entre os temas sugeridos para inclusão no programa de mentoria em 2021, destacou-se educação financeira, tópico citado por $35 \%$ dos residentes $(n=14)$. Citaram-se ainda "como definir sua subespecialidade", "relação interpessoal e liderança", "relação interpessoal com a equipe multidisciplinar", "avaliação da experiência do paciente" e "papel do médico residente no enfrentamento da pandemia".

Tabela 1. Quais dos temas discutidos tiveram maior potencial de mudança na sua atuação como profissional de saúde?

\begin{tabular}{cc}
\hline Temáticas relevantes & $\%(4-5)$ \\
\hline Burnout e qualidade de vida & $77,8 \%$ \\
Empatia & $70,3 \%$ \\
Gestão de conflitos & $66,6 \%$ \\
Profissionalismo & $59,3 \%$ \\
Gestão de tempo & $51,9 \%$ \\
Role-model & $48,1 \%$ \\
\hline
\end{tabular}


Pontos de melhoria foram solicitados aos mentorados, sendo pontuado que "Mudar a turma do mentoring mais de uma vez, para termos contato com mais preceptores e outros colegas" seria algo positivo, bem como receber "Residentes de outras especialidades, para avaliar a visão de cada especialidade sobre estes pontos", como forma de agregar e diversificar a visão dos temas propostos. Além disso, como não havia consenso sobre o horário de realização das reuniões, o que foi considerado um aspecto negativo, sugeriu-se que a atividade fosse "marcada com mais antecedência". Uma outra sugestão referiu-se à possibilidade de "enquadrar [a atividade] dentro das 60 horas de carga horária da residência", uma vez que alguns mentores e mentorados realizavam as reuniões em horários que extrapolavam a carga horária preconizada.

\section{DISCUSSÃO}

Ao avaliarmos o feedback dos mentorados a respeito da estruturação de temas e da atividade em si, bem como sobre a relevância prática do que se foi discutido, demonstramos que há um impacto positivo na formação do médico residente. Ao abordarmos temas não usualmente discutidos na formação acadêmica e que, invariavelmente, fazem parte da vida de um profissional formado, tivemos uma percepção de que há mudança de conduta no campo de prática, gerando reflexões benéficas ${ }^{5}$.

Programas de mentoria foram relacionados à melhora da autoconfiança, com impacto na carreira profissional em longo prazo, havendo relatos, em estudos observacionais realizados na Inglaterra, de melhora de resultados nas provas anuais de avaliação de desempenho e progressão de competências, com $86,4 \%$ desses mentorados atingindo nota máxima de forma significativa ${ }^{5}$.

Além de desempenho, percebemos que o tema de maior relevância para os residentes do programa foi burnout e qualidade de vida. Há uma prevalência de cerca de $40 \%$ da síndrome do esgotamento profissional entre médicos e residentes, tendo consequências negativas para a saúde mental desses profissionais. A residência médica, por sua estrutura e natureza de funcionamento, eleva o risco de problemas mentais relacionados a essa síndrome ${ }^{6,8}$. Dessa forma, com a associação de programas de mentoria à possibilidade de suporte psicossocial, com criação de um ambiente de livre acesso entre residente e preceptor ${ }^{4}$, percebemos a importância da atividade no contexto de um jovem médico dentro da sua especialidade, como citou um dos residentes: "Falar sobre e ouvir os conflitos dos outros nos ensina a abordar melhor esses problemas no dia a dia".

A mentoria, ainda durante a residência médica, promove o desenvolvimento de profissionalismo ${ }^{4,7}$. Em uma pesquisa de satisfação realizada em um programa de mentoria norte-americano para residentes de clínica médica, 70\% dos participantes relataram que o mentoring foi importante para o seu desenvolvimento profissional ${ }^{4}$, resultado semelhante ao visualizado no nosso questionário².

Segundo o relato de Ramanan et al. ${ }^{4}$, quando questionados sobre as dificuldades que poderiam ser encontradas por residentes que não faziam parte de programas de mentoria, 37\% dos residentes referiram ter medo de se aproximar de algum preceptor para tratar-se de temas discutidos na mentoria, enquanto $28 \%$ relataram que não havia ninguém em quem pudessem confiar ${ }^{5,10}$. No entanto, quanto aos mentorados, 52\% afirmaram ter uma relação pessoal significativa com o seu mentor ${ }^{4}$. Dessa forma, um dos aspectos positivos da atividade é justamente promover a aproximação entre aprendiz e professor, uma vez que a medicina, historicamente, é ensinada pelo profissional mais experiente. Na nossa avaliação e baseados nas respostas ao nosso questionário, entendemos que houve um vínculo positivo entre mentores e mentorados ${ }^{5,9}$.

Tivemos algumas limitações na realização da atividade, como a dificuldade para definir, de forma consensual, os horários das reuniões, que ocorriam, em diversos momentos, no período noturno, extrapolando o que era preconizado como carga horária ideal para a residência. Além disso, a heterogeneidade de alguns grupos, como citado no nosso questionário, pode ser resultado da dificuldade de padronização na introdução da discussão dos temas pelos mentores, sendo um ponto a ser melhorado para as próximas turmas durante a capacitação.

\section{CONCLUSÃO}

No Ceará, o programa de mentoring para os residentes de clínica médica foi inédito. Conforme as respostas do questionário, constatamos um efeito positivo do programa nos mentorados, em que temas igualmente importantes para a educação médica foram discutidos com mais profundidade, possibilitando um maior crescimento pessoal e profissional. Diante dessa experiência, os autores sugerem que o mentoring seja mantido e incluído como atividade essencial no currículo dos programas de residência médica.

\section{CONTRIBUIÇÃO DOS AUTORES}

Fernando Lima Lopes Filho participou da redação do manuscrito, da edição e formatação do artigo, e da coleta de dados do questionário. Francisco Vileimar Andrade de Azevedo participou da revisão do artigo e da elaboração do questionário. Kristopherson Lustosa Augusto participou do design e da revisão do artigo. Hyvinna Suellen de Oliveira Silveira participou da formatação do artigo. Fabrício André Martins da Costa 
participou da concepção do projeto e da revisão do artigo. Karine Paschoal Botelho Alves participou da edição do texto, do fornecimento e da orientação das referências, e da revisão do artigo.

\section{CONFLITO DE INTERESSES}

Declaramos não haver conflito de interesses.

\section{FINANCIAMENTO}

Declaramos não haver financiamento.

\section{REFERÊNCIAS}

1. Moreira SNT, Albuquerque ICS, Pinto Junior FEL, Gomes AHB. Programa de Mentoria do curso de Medicina da Universidade Federal do Rio Grande do Norte: atividades integrativas em foco. Rev Bras Educ Med. 2020;44(4):e169. doi: 10.1590/1981-5271v44.4-20200103.

2. Akinla $O$, Hagan $P$, Atiomo W. A systematic review of the literature describing the outcomes of near-peer mentoring programs for first year medical students. BMC Med Educ. 2018.18(1):98. doi: 10.1186/s12909018-1195-1.

3. Henry-Noel N, Bishop M, Gwede CK, Petkova E, Szumacher E. Mentorship in medicine and other health professions. J Cancer Educ. 2019;34(4):62937. doi: 10.1007/s13187-018-1360-6.
4. Ramanan RA, Phillips RS, Davis RB, Taylor WC. Mentoring matters. Mentoring and career preparation in internal medicine residency training. $J$ Gen Intern Med. 2006;21(4):340-5. doi: 10.1111/j.1525-1497.2006.00346.x.

5. Ong J, Swift C, Magill N, Ong S, Day A, Al-Naeeb Y, et al. The association between mentoring and training outcomes in junior doctors in medicine: an observational study. BMJ Open. 2018;8(9):e020721. doi: 10.1136/ bmjopen-2017-020721.

6. Dyrbye LN, Burke SE, Hardeman RR, Herrin J, Wittlin NM, Yeazel M, et al. Association of clini-cal specialty with symptoms of burnout and regret among US resident physicians. JAMA Netw Open. 2018;320(11):1114-30. doi: 10.1001/jama.2018.12615.

7. Ortega G, Smith C, Pichardo MS, Ramirez A, Soto-Greene M, Sánchez JP. Preparing for an academic career: the significance of mentoring. MedEdPORTAL. 2018;14:10690. doi: 10.15766/mep_2374-8265.10690.

8. Moreira SNT, Vasconcellos RLSS, Heath N. Estresse na formação médica: como lidar com essa realidade? Rev Bras Educ Med. 2015;39(4):558-64. doi: 10.1590/1981-52712015v39n4e03072014.

9. Martins AF, Bellodi PL. Mentoring: uma vivência de humanização e desenvolvimento no curso médico. Interface Comun Saúde Educ. 2016;20(58):715-26. doi: 10.1590/1807-57622015.0432.

10. Cameron D, Dromerick LJ, Ahn J, Dromerick AW. Executive/life coaching for first year medi-cal students: a prospective study. BMC Med Educ. 2019;19(1):163. doi: 10.1186/s12909-019-1564-4. 